\title{
Percutaneous endoscopic lumbar discectomy utilizing ventral epiduroscopic observation technique and foraminoplasty for transligamentous extruded nucleus pulposus: technical note
}

\author{
Tatsuhiko Henmi, MD, ${ }^{1}$ Tomoya Terai, MD, PhD,1 Naohito Hibino, MD, PhD, ${ }^{1}$ Shinji Yoshioka, MD,1 \\ Kenji Kondo, MD, ${ }^{1}$ Yuichiro Goda, MD, ${ }^{2}$ Fumitake Tezuka, MD, ${ }^{2}$ and Koichi Sairyo, MD, PhD ${ }^{2}$ \\ 1Department of Orthopedic Surgery, Tokushima Prefecture Naruto Hospital; and 2Department of Orthopedics, \\ Tokushima University, Tokushima, Japan
}

\begin{abstract}
Percutaneous endoscopic discectomy (PED) is a minimally invasive disc surgery that can be performed under local anesthesia and requires only an 8-mm skin incision. For transligamentous extruded nucleus pulposus with foraminal stenosis, it is very hard to remove the migrated mass with a simple transforaminal approach. For such difficult cases, foraminoplasty and an epiduroscopic technique is useful. A 29-year-old man visited the authors' hospital, complaining of low-back and right leg pain. MRI revealed a massive herniated nucleus pulposus with foraminal stenosis. A transforaminal PED was planned to remove the herniated mass. Through the inside-out technique, the base of the herniated mass was removed. Following the foraminoplasty, the cannula was moved into the epidural space. With epidural observation just beneath the nerve root, the extruded transligamentous fragment was confirmed and removed en bloc. Immediately after the surgery, the patient's symptoms resolved. The combination of foraminoplasty and epiduroscopic observation during the transforaminal approach for PED is a useful and reliable technique to remove extruded transligamentous disc fragments.
\end{abstract}

http://thejns.org/doi/abs/10.3171/2015.4.SPINE141305

KEY WORDS percutaneous endoscopic lumbar discectomy; herniated nucleus pulposus; foraminoplasty; epidural observation

$\mathrm{P}$ ERCUTANEOUS discectomy for herniated nucleus pulposus (HNP) was developed by Hijikata. ${ }^{6}$ The technique is minimally invasive; however, under image intensifier guidance alone, percutaneous discectomy is not reliable for removal of the extruded fragment. To overcome such shortcomings in the percutaneous discectomy procedure, percutaneous endoscopic discectomy (PED) was developed. After several trials,, 919 the current system of single-portal endoscopic discectomy was developed in the late 1990s. ${ }^{20-22}$ After 1990, the technique was further developed by utilizing a spinal endoscope and a tubular retractor. This endoscope-assisted transtubular surgery was eventually established by Foley and Smith ${ }^{5}$ and Destandeau ${ }^{2}$ in what is now known as microendoscopic discectomy (MED). Surgeons can access the HNP through the interlaminar space. This surgical approach to HNP is removal, which includes laminotomy, partial removal of the ligamentum flavum, and HNP fragment removal, in a procedure known as Love's technique. An extended musclesplitting dissection causes postoperative low-back pain. The benefit of transforaminal approach is that it is conducted under local anesthesia with minimal invasiveness. The use of a spinal endoscope improves reliability when removing extruded disc fragments. Recently, Dezawa and colleagues ${ }^{3,4,10,16}$ established an advanced technique using a high-speed drill that enables most HNPs to be removed using a minimally invasive PED system.

To avoid surgery-related complications,${ }^{17}$ the inside-out transforaminal technique through the safety triangle is recommended. ${ }^{15}$ Through this technique, contained HNP,

ABBREVIATIONS HNP = herniated nucleus pulposus; $M E D=$ microendoscopic discectomy; $\mathrm{PED}=$ percutaneous endoscopic discectomy.

SUBMITTED December 22, 2014. ACCEPTED April 22, 2015.

INCLUDE WHEN CITING Published online October 16, 2015; DOI: 10.3171/2015.4.SPINE141305. 
such as protrusion or subligamentous extrusion, is easy to remove. On the other hand, in some cases of noncontained HNP, such as transligamentous extrusion into the epidural space or sequestration, it is difficult to remove the HNP in the epidural space. For such HNPs, other techniques have been reported previously., ${ }^{3,411}$ Among them, the epiduroscopic technique ${ }^{11}$ can be done after administration of local anesthesia, as this technique is a modification of the transforaminal technique. During the epiduroscopic technique, the 8-mm cannula must be placed in the intervertebral foramen. Exiting nerve root injury is likely to occur in cases with foraminal stenosis. ${ }^{17}$ Therefore, to safely insert the cannula with the endoscope into the epidural space through the intervertebral foramen in patients with a foraminal stenosis, a foraminoplasty technique can be used. ${ }^{12}$ Here, we report a case of HNP with foraminal stenosis in which the HNP was successfully removed with a combination of foraminoplasty ${ }^{12}$ and the epiduroscopic technique. ${ }^{11}$

\section{Case Report}

A 29-year-old man, a medical doctor and general surgeon, had been experiencing low-back pain and right leg pain for 4 months. He was diagnosed as having an HNP of the lumbar spine at a nearby hospital. He was prescribed analgesics, but his symptoms did not improve. He consulted our institution, seeking minimally invasive surgery for symptomatic relief because, as a physician, he could not take a long sick leave. At the first visit, his visual analog scale scores for low-back pain and leg pain were 8/10 and $6 / 10$, respectively. Neurological examination revealed muscle weakness; the strength of the right tibialis anterior was $4 / 5$, and that of the right extensor hallucis longus was $2 / 5$. Sensory disturbance was noted along the right lower leg and right big toe. The straight leg raise test of the right leg was positive at $60^{\circ}$. Laboratory data showed no inflammatory signs. Selective nerve root block at the right L-5 nerve root achieved temporary pain relief. Our clinical diagnosis was L-5 nerve root radiculopathy.

Figure 1 shows the MR images at presentation. HNP at L4-5 is evident on the sagittal scan (Fig. 1 left). The size of the mass on the axial image through L4-5 was about $50 \%$ of the canal (Fig. 1 right). CT with discogra-
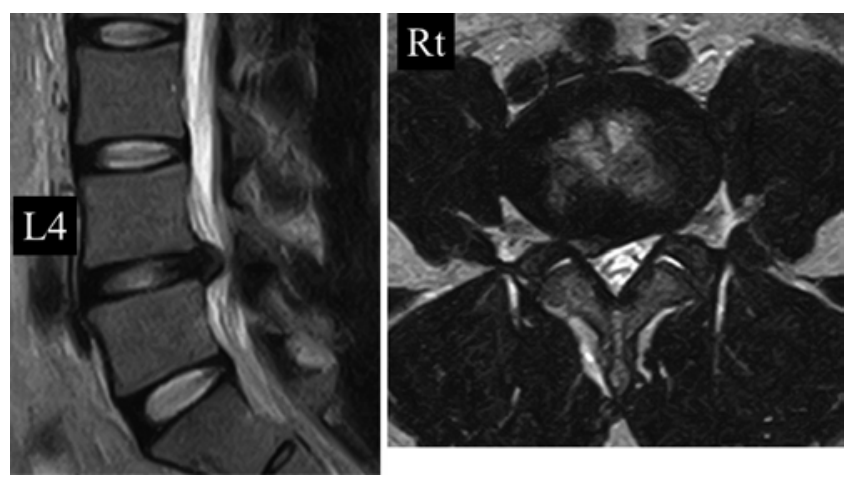

FIG. 1. T2-weighted MR images obtained at presentation. The HNP at L4-5 is evident on the right midsagittal scan (left), and the size of the mass on the axial image through L4-5 is about $50 \%$ of the canal (right).
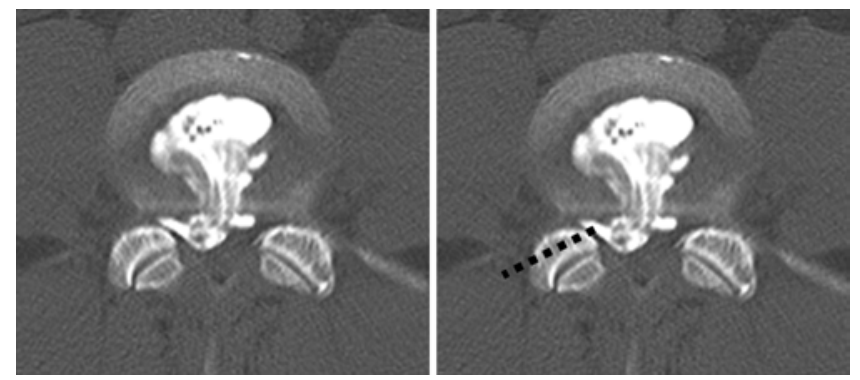

FIG. 2. CT scans with discography. Left: The nucleus pulposus extruded through an annular tear in the midline of the disc. Right: The dotted line indicates the planned resection area by foraminoplasty.

phy revealed that the nucleus pulposus extruded through an annular tear in the midline of the disc (Fig. 2 left). PED via a transforaminal approach was planned to remove the herniated mass. As shown in Fig. 2 left, the facet joint was too large to access the migrated HNP with the transforaminal technique, so we also planned foraminoplasty (Fig. 2 right).

Minimally invasive removal of the HNP was planned using a transforaminal technique with a percutaneous endoscope. Under local anesthesia, an 8-mm skin incision was made $10 \mathrm{~cm}$ lateral to the midline. After the dilating maneuver, a beveled cannula was inserted into the posterior disc space through the intervertebral foramen. Using the inside-out technique, the base of the herniated mass was first removed. Then the cannula was moved to the subligamentous space, and the fragments were removed. Next, foraminoplasty was performed, widening the intervertebral foramen, prior to epiduroscopic removal of the migrated HNP. The hypertrophied inferior facet joint in Fig. 3 left was noted, and it was removed using a highspeed drill. Figure 3 right shows the enlarged foramen. The cannula was then inserted into the epidural space. Figure 4 shows how the cannula was moved from the intradiscal space to the ventral epidural space. Figure 5 left indicates the location of the cannula on the $\mathrm{C}$-arm image intensifier. We use freehand, biplane, and C-arm-guided access and an optimal trajectory, oriented by endoscopic landmarks. The radiation exposure time has not yet been estimated. However, the radiation time would be decreased with the
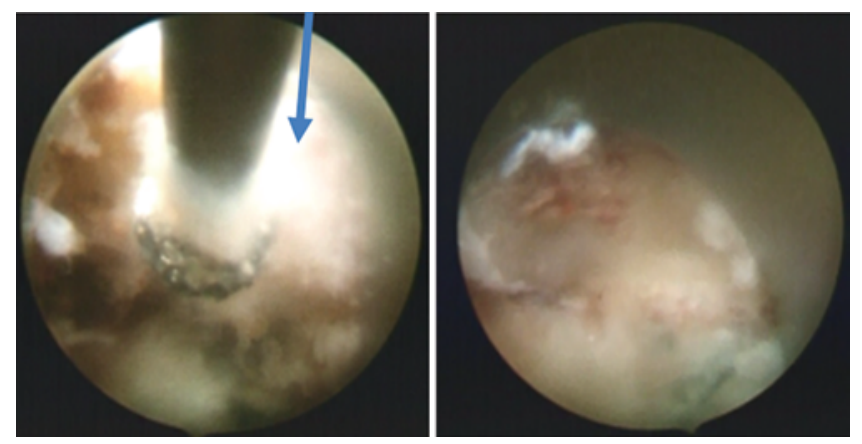

FIG. 3. Endoscopic view at the foramen before (left) and after (right) foraminoplasty. The hypertrophied inferior facet joint (left) was removed with the high-speed drill. The enlarged foramen (right). Figure is available in color online only. 


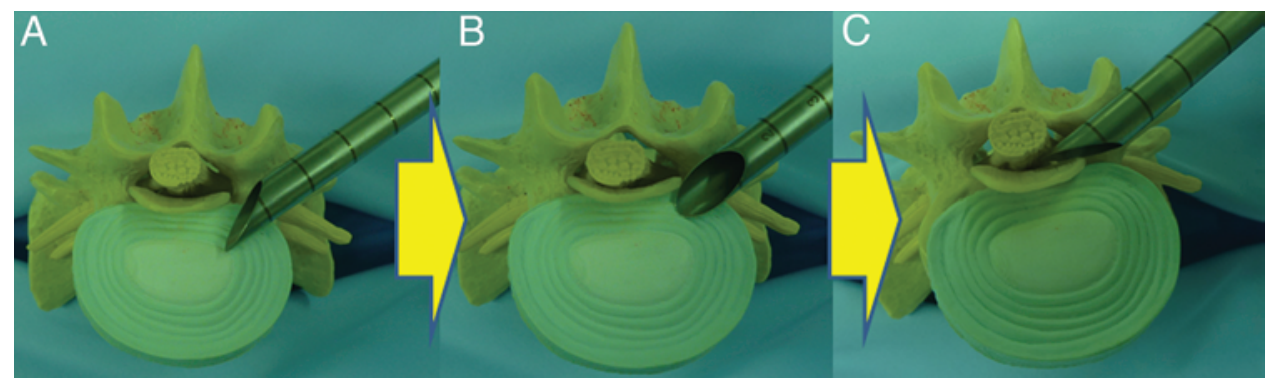

FIG. 4. Rotation maneuver of the cannula (B) from the intradiscal space (A) to the ventral epidural space (C). Figure is available in color online only.

clinical experience. The longer side of the beveled cannula was placed into the dorsal aspect to protect the nerve root and dura from injury. Figure 5 right shows a schema of the cannula in the epidural space. The endoscope is in the ventral epidural space; this is the epiduroscopic observation technique (Video 1).

VIDEO 1. Foraminoplasty was performed. The hypertrophied inferior facet joint was removed using a high-speed drill. The cannula was then inserted into the epidural space. The longer side of the beveled cannula was placed to the dorsal aspect to protect the nerve root and dura. The HNP fragments were removed. Copyright Koichi Sairyo. Published with permission. Click here to view.

As shown in Fig. 6, the surface of the disc and herniated mass can be observed from the epiduroscope. The mass was completely removed en bloc (Fig. 7 left). Throughout the epiduroscopic procedure, the nerve root and dura mater were protected by the longer wall of the beveled cannula. Figure 7 right shows the HNP removed in the procedure. The operative time was 75 minutes, and the estimated blood loss was negligible. Immediately after the operation, the patient's leg pain resolved and the straight leg raise test was negative at $70^{\circ}$. As in a previous report, ${ }^{17}$ we encountered no surgery-related complication, such as nerve root injury, dural tear, hematoma, headache, or neck pain.

After surgery, the patient's symptoms dramatically improved. Visual analog scale scores for low-back and leg pain improved to $0 / 10$. On postoperative Day 5 , he returned to work as a general surgeon without any symp-
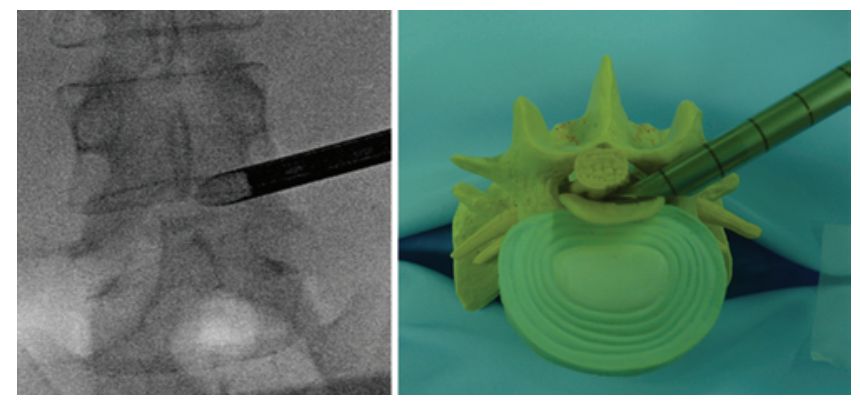

FIG. 5. Location of the cannula in the ventral epiduroscopic procedure. Left: $O n$ the $\mathrm{C}$-arm image, the tip of the cannula is inserted at the central portion in the canal. In this situation, the longer side of the beveled cannula is placed to the dorsal aspect to protect the nerve root and dura from injury. Right: The schema of the cannula in the epidural space. Figure is available in color online only. toms. Three months after surgery, the patient's visual ana$\log$ scale scores for low-back pain and leg pain were $0 / 10$ and 0/10, respectively. His Japanese Orthopaedic Association score improved to 29/29 points from 11/29.

On postoperative Day 2 and at 3 months, sagittal and axial MR images showed that the HNPs were successfully removed (Fig. 8). Figure 9 shows pre- and postoperative $\mathrm{CT}$ scans. Note the enlargement of the intervertebral foramen after foraminoplasty. Figure 10 indicates the location of the skin incision.

\section{Discussion}

The great efforts by Yeung and colleagues ${ }^{20-22}$ established the current system of transforaminal PED. In Japan, Dezawa and colleagues $3,4,6,10,15-17,21,22$ developed new devices for PED, such as an ultrathin high-speed drill and contributed to further development of the PED procedure. In the present case, by using a simple transforaminal approach, it was not difficult to remove the transligamentous

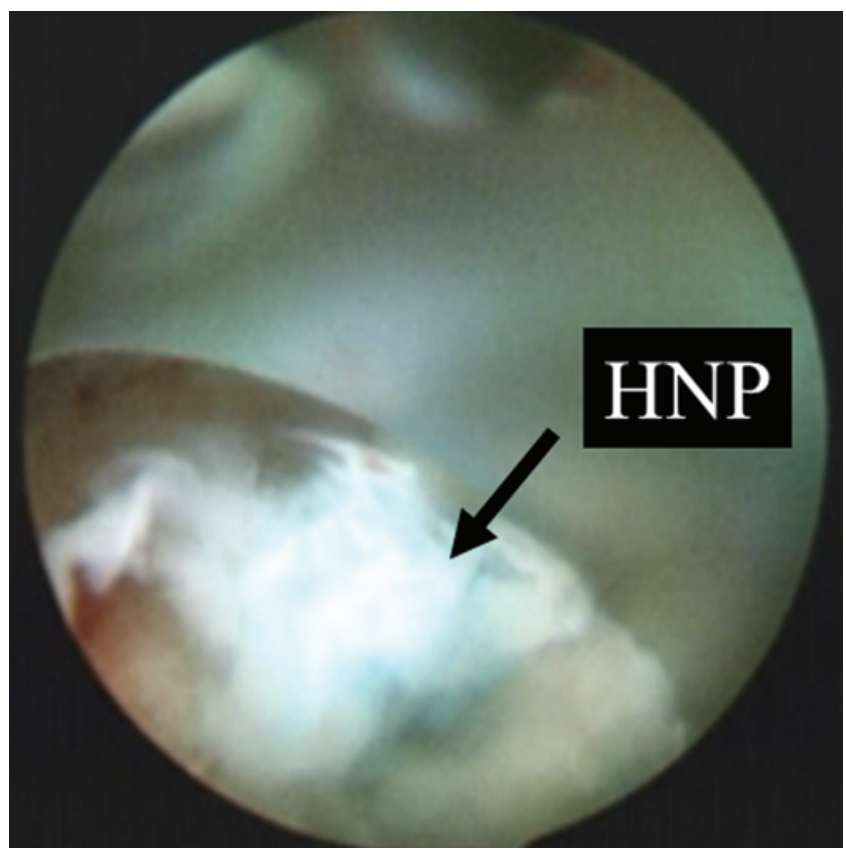

FIG. 6. Ventral epiduroscopic observation view. The herniated mass is stained blue and is clearly visible. Figure is available in color online only. 


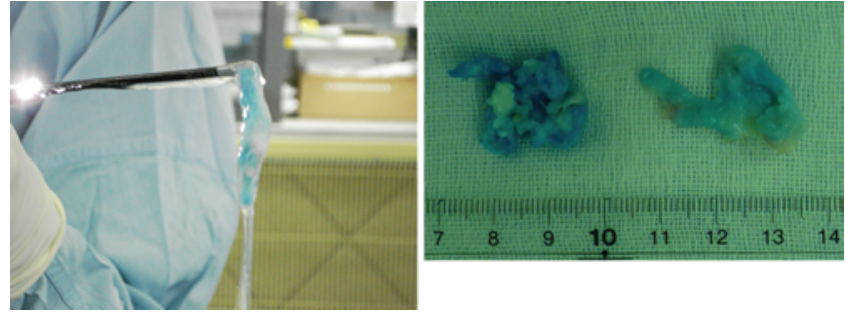

FIG. 7. Endoscopic removal of the migrated disc fragments. Left: The transligamentous extruded mass is completely removed en bloc. Right: The mass on the right is a single fragment from the ventral epidural space, and the mass on the left is the multiple fragments removed piece by piece from the annular tear and subligamentous space. Figure is available in color online only.

extruded HNP in the epidural space. We used 2 additional techniques to remove the migrated fragment in the epidural space: epiduroscopic observation ${ }^{11}$ and foraminoplasty. ${ }^{12}$

Endoscopic discectomy via a percutaneous transforaminal posterolateral approach is an alternative technique used to treat lumbar disc herniations. The advantages of this technique include less paraspinal musculature trauma and smaller wounds. Bone removal is not required to decompress the exiting nerve root, and this avoids the risk of inducing spinal instability. ${ }^{7-9,18}$ Birkenmaier et al. ${ }^{1}$ reviewed 5 comparative studies of the present technique and conventional open surgery and concluded that PED had
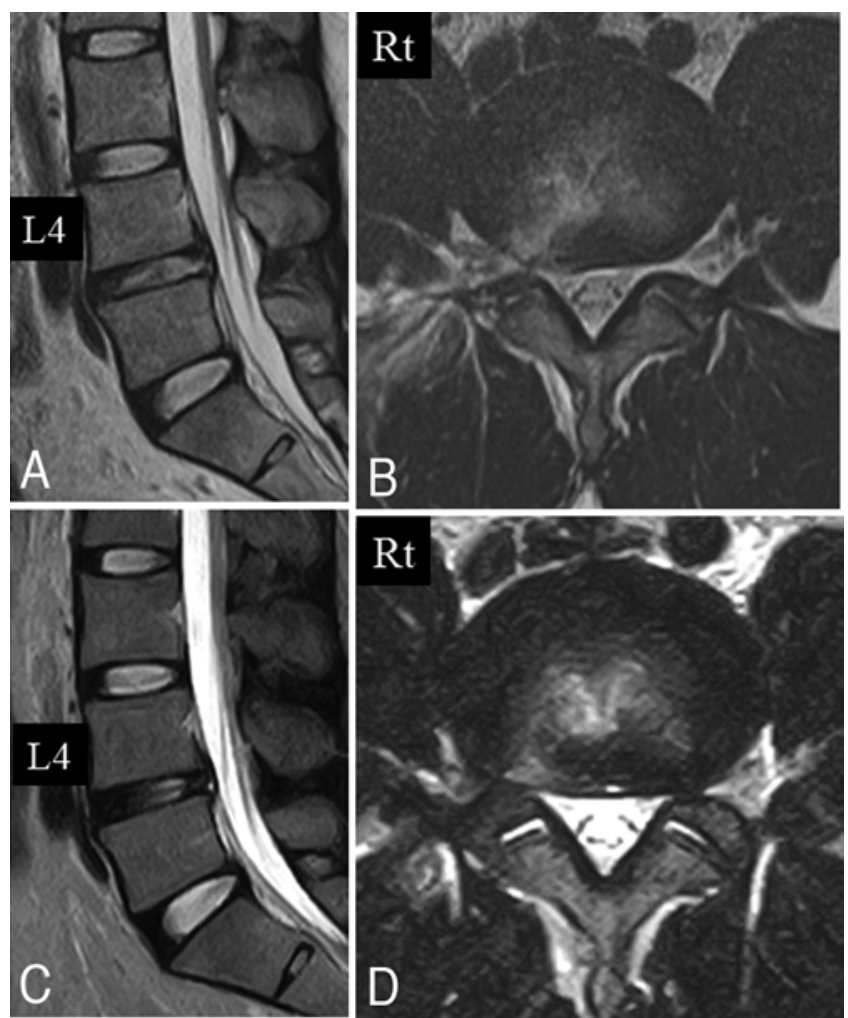

FIG. 8. MR images on postoperative Day 2 (A and B) and at 3 months ( $C$ and $D)$. Sagittal ( $A$ and $C$ ) and axial $(B$ and $D)$ views showing that the HNPs were successfully removed.
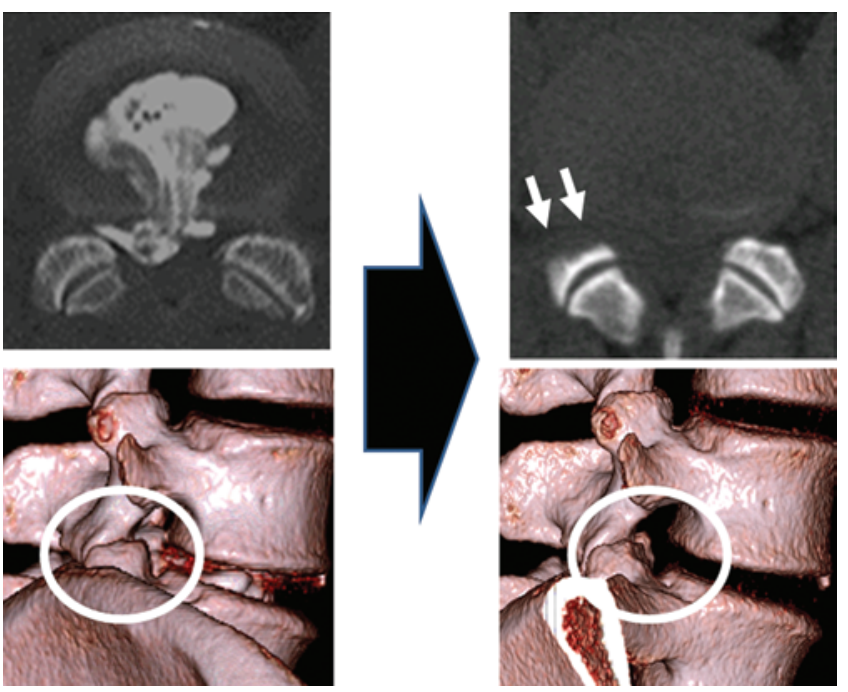

FIG. 9. Preoperative (left) and postoperative (right) CT scans. Note the enlargement of the intervertebral foramen after foraminoplasty (white arrows). Figure is available in color online only.

similar clinical outcomes. Moreover, they emphasized the following benefits of PED: shorter operating time, lower estimated blood loss during surgery, fewer surgery-related complications, less surgical site pain immediately after surgery, less need for postoperative pain medication, shorter hospital stay, and a faster return to work. The greatest benefit of this PED procedure is that it can be done under local anesthesia.

\section{Epiduroscopic Observation}

As shown in Fig. 4A, a contained HNP is a good indication for removal by a simple transforaminal approach with the inside-out technique. However, in some cases of noncontained HNP, such as transligamentous, extruded, or sequestration types, complete removal is difficult. In such situations, we need additional techniques. The interlaminar approach ${ }^{4}$ and translaminar technique ${ }^{3}$ are good options for this purpose but cannot be performed with local anesthesia. The transforaminal epiduroscopic observation technique is the other option. It can be conducted
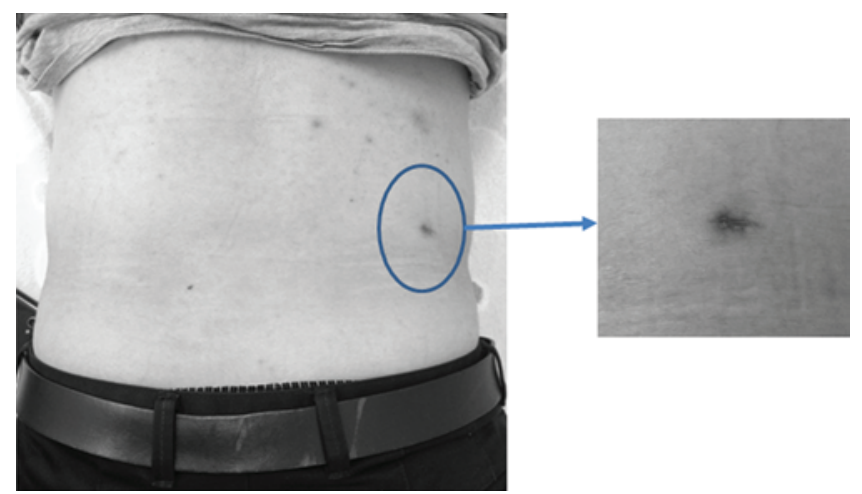

FIG. 10. The surgical scar is $8 \mathrm{~mm}$. Figure is available in color online only. 
under local anesthesia and performed via a transforaminal approach.

Figure 5 right shows a schema of the epiduroscopic observation technique used in this case. Using this technique, one can observe the herniated fragments from the ventral epidural space. Indeed, as shown in Fig. 6, in our case an uncontained HNP that had migrated into the epidural space could be clearly seen by seeking the fragments stained blue with indigo carmine. A beveled cannula is commonly used for the transforaminal PED procedure. During the inside-out technique, the bevel aspect faces upward (Fig. 4A), whereas during the epiduroscopic observation it faces downward (Fig. 4C). The longer wall of the cannula is placed against the neural tissue, including the traversing nerve root and dura mater. This technique enables observation of the central portion of the spinal canal (Fig. 5). Too much retraction of neural tissue may cause palsy. In the literature, many PED-related complications have been reported. Among them, exiting nerve root injury is a particular complication in the transforaminal approach. When nerve damage by the cannula occurs when the patient is under general anesthesia, the patient does not feel any pain. The advantage of local anesthesia is that this kind of complication can be avoided. The most beneficial aspect of the transforaminal epiduroscopic observation procedure is that it can be conducted under local anesthesia. During the operation, the patient may feel pain and numbness and can complain if any develops. Thus, this technique is safe and reliable for removal of migrated fragments in the epidural space in noncontained herniations with local anesthesia. However, there is one limitation in the narrow foramen case. If the 8-mm cannula is inserted through the narrow foramen, the cannula may compress the exiting nerve root, leading to palsy. For such cases, foraminoplasty is necessary to safely insert the cannula into the epidural space.

\section{Foraminoplasty}

As shown in Fig. 2, we planned foraminoplasty to safely insert the cannula into the epidural space. Figure 3 right shows the enlarged foramen following the foraminoplasty. Following enlargement of the foramen, the cannula could be inserted without compressing the exiting nerve root (Fig. 5 left). Lee et al. ${ }^{12}$ showed that foraminoplasty is essential to avoid injuring the exiting nerve root by insertion of the cannula through the narrow foramen. As in the present case, partial removal of the inferior facet is often useful; however, in severely narrow cases, pediculotomy is also necessary.

The literature on the epiduroscopic technique with transforaminal approach is sparse. ${ }^{11,13,14}$ These articles only mention that the cannula was placed at the ventral epidural space to remove the migrated fragment, and the technical details are unclear. Ruetten et al. ${ }^{14}$ mentioned the importance of the additional foraminoplasty for the safety of ventral epiduroscopic observation, which would be very similar procedurally to our case. However, the exact technique is not well described. Here, we have clearly demonstrated the technical procedure for epiduroscopic transforaminal removal of HNP following foraminoplasty using the endoscope.
In conclusion, the combination of foraminoplasty and epiduroscopic observation during the transforaminal approach for PED is a useful and reliable technique to remove transligamentous extruded disc fragments.

\section{References}

1. Birkenmaier C, Komp M, Leu HF, Wegener B, Ruetten $\mathrm{S}$ : The current state of endoscopic disc surgery: review of controlled studies comparing full-endoscopic procedures for disc herniations to standard procedures. Pain Physician 16:335-344, 2013

2. Destandau J: [Technical features of endoscopic surgery for lumbar disc herniation: 191 patients.] Neurochirurgie 50:610, 2004 (Fr)

3. Dezawa A, Mikami H, Sairyo K: Percutaneous endoscopic translaminar approach for herniated nucleus pulposus in the hidden zone of the lumbar spine. Asian J Endosc Surg 5:200-203, 2012

4. Dezawa A, Sairyo K: New minimally invasive discectomy technique through the interlaminar space using a percutaneous endoscope. Asian J Endosc Surg 4:94-98, 2011

5. Foley KT, Smith MM: Microendoscopic discectomy. Tech Neurosurg 3:301-307, 1997

6. Hijikata S: Percutaneous nucleotomy. A new concept technique and 12 years' experience. Clin Orthop Relat Res 238:9-23, 1989

7. Kambin P, Brager MD: Percutaneous posterolateral discectomy. Anatomy and mechanism. Clin Orthop Relat Res 223:145-154, 1987

8. Kambin P, Sampson S: Posterolateral percutaneous suctionexcision of herniated lumbar intervertebral discs. Report of interim results. Clin Orthop Relat Res (207):37-43, 1986

9. Kambin P, Schaffer JL: Percutaneous lumbar discectomy. Review of 100 patients and current practice. Clin Orthop Relat Res (238):24-34, 1989

10. Kitahama Y, Sairyo K, Dezawa A: Percutaneous endoscopic transforaminal approach to decompress the lateral recess in an elderly patient with spinal canal stenosis, herniated nucleus pulposus and pulmonary comorbidities. Asian J Endosc Surg 6:130-133, 2013

11. Lee S, Kim SK, Lee SH, Kim WJ, Choi WC, Choi G, et al: Percutaneous endoscopic lumbar discectomy for migrated disc herniation: classification of disc migration and surgical approaches. Eur Spine J 16:431-437, 2007

12. Lee SH, Kang HS, Choi G, Kong BJ, Ahn Y, Kim JS, et al: Foraminoplastic ventral epidural approach for removal of extruded herniated fragment at the L5-S1 level. Neurol Med Chir (Tokyo) 50:1074-1078, 2010

13. Ruetten S, Komp M, Godolias G: An extreme lateral access for the surgery of lumbar disc herniations inside the spinal canal using the full-endoscopic uniportal transforaminal approach-technique and prospective results of 463 patients. Spine (Phila Pa 1976) 30:2570-2578, 2005

14. Ruetten S, Komp M, Merk H, Godolias G: Use of newly developed instruments and endoscopes: full-endoscopic resection of lumbar disc herniations via the interlaminar and lateral transforaminal approach. J Neurosurg Spine 6:521-530, 2007

15. Sairyo K, Egawa H, Matsuura T, Takahashi M, Higashino K, Sakai T, et al: State of the art: Transforaminal approach for percutaneous endoscopic lumbar discectomy under local anesthesia. J Med Invest 61:217-225, 2014

16. Sairyo K, Kitagawa Y, Dezawa A: Percutaneous endoscopic discectomy and thermal annuloplasty for professional athletes. Asian J Endosc Surg 6:292-297, 2013

17. Sairyo K, Matsuura T, Higashino K, Sakai T, Takata Y, Goda $\mathrm{Y}$, et al: Surgery related complications in percutaneous en- 
doscopic lumbar discectomy under local anesthesia. J Med Invest 61:264-269, 2014

18. Schaffer JL, Kambin P: Percutaneous posterolateral lumbar discectomy and decompression with a $6.9 \mathrm{~mm}$ cannula: Analysis of operative failures and complications. J Bone Joint Surg Am 73:822-831, 1991

19. Schreiber A, Leu H: Percutaneous nucleotomy: technique with discoscopy. Orthopedics 14:439-444, 1991

20. Yeung AT: The evolution of percutaneous spinal endoscopy and discectomy: state of the art. Mt Sinai J Med 67:327332,2000

21. Yeung AT, Tsou PM: Posterolateral endoscopic excision for lumbar disc herniation: surgical technique, outcome, and complications in 307 consecutive cases. Spine (Phila Pa 1976) 27:722-731, 2002

22. Yeung AT, Yeung CA: Minimally invasive techniques for the management of lumbar disc herniation. Orthop Clin North Am 38:363-372, vi, 2007

\section{Disclosure}

The authors report no conflict of interest concerning the materi- als or methods used in this study or the findings specified in this paper.

\section{Author Contributions}

Conception and design: Henmi. Acquisition of data: Terai. Analysis and interpretation of data: Terai. Drafting the article: Sairyo. Critically revising the article: Terai. Reviewed submitted version of manuscript: Hibino, Yoshioka, Kondo, Goda, Tezuka. Study supervision: Sairyo.

\section{Supplemental Information \\ Video}

Video 1. https://vimeo.com/140800256.

\section{Correspondence}

Koichi Sairyo, Department of Orthopedics, Tokushima University, 3-18-15 Kuramoto, Tokushima 770-8503, Japan. email: sairyokun@hotmail.com. 